\title{
EDITORIAL
}

\section{A special issue on Light-Emitting Diodes}

\author{
Xiaobing LUO $(\bowtie)^{1,2}$, Sheng LIU ${ }^{2,3}$ \\ 1 School of Energy and Power Engineering, Huazhong University of Science and Technology, Wuhan 430074, China \\ 2 Wuhan National Laboratory for Optoelectronics, Huazhong University of Science and Technology, Wuhan 430074, China \\ 3 School of Mechanical Science and Engineering, Huazhong University of Science and Technology, Wuhan 430074, China
}

(C) Higher Education Press and Springer-Verlag Berlin Heidelberg 2012

As an emerging and energy efficient lighting technology, light-emitting diode (LED) based solid state lighting is expected to significantly reduce global energy consumption and green-house emission for its extraordinary characteristics compared with traditional light sources, including high luminous efficiency, small size, potentially high device and system reliability, long lifetime, etc. Since the LEDs are unable to be applied before being packaged, the packaging technologies are critically needed in terms of materials selection, process development, co-design with multi-physics consideration, integrated consideration of chip and packaging design. Packaging is therefore essential in enhancing the desired long-term reliability and realizing the full potentials of optical performance of LED devices, which still needs us to conduct more fundamental research. Over the decades, many novel packaging technologies have been proposed and some of them have been commercially applied, yet some of them are still under development.

To further improve the optical performance and reliability of the LED packages from the view of manufacturing, cost and application, the efforts are made in the whole LED packaging processes from the chip level to the application level. Any progress of LED packaging technologies will benefit the manufacturers, researchers, and of course the final users. Therefore, it is our intention to bring the research community's attention to these particular research areas. In this "Special Issue on Light-Emitting Diodes", 1 review article and 5 research articles focusing on relevant subjects by internationally active groups in the field are specially presented.

More specifically, Prof. Lee of Hong Kong University of Science and Technology reviewed the advanced LED wafer level packaging technologies and covered some key packaging processes. Prof. Kim from Korea Institute of Ceramic Engineering and Technology suggested a new design and material processing technology to realize price and performance advantageous LED packaging solution. Prof. Rao from University of Electronic Science and Technology of China proposed a self-adaptive phosphor coating technology to further improve the quality of phosphor coating layer on the LED chips. Prof. Jang from Yeungnam University demonstrated the high-performance and current crowding-free InGaN/GaN LEDs using an electrically-reverse-connected Schottky diode and an Mgdelta doped layer. Prof. Luo from Huazhong University of Science and Technology studied the phosphor coating process by calculating the optical constants of phosphor layer and by investigating the effects of phosphor surface geometry on the optical performance of LEDs, respectively.

Overall, articles in this special issue exhibit the good achievements of LED, particularly in the interests about the LED packaging technologies for better optical performance, better manufacturing processes and better applications. We hope they are helpful to the LED industry and researchers.

Finally, we would like to give our acknowledgements to authors to present their work and to reviewers for their valuable comments.

Received February 14, 2012

E-mail: luoxb@mail.hust.edu.cn 

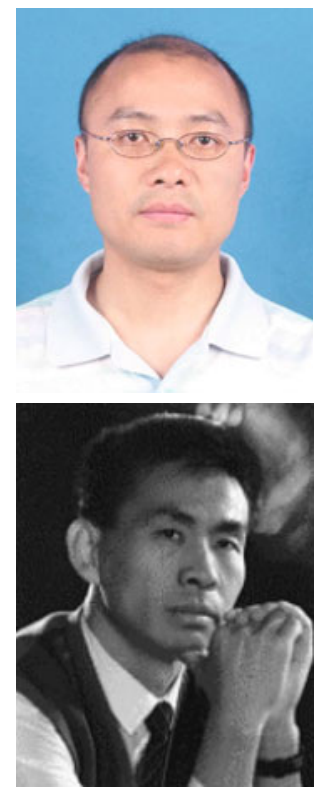

Xiaobing Luo is a professor in Huazhong University of Science and Technology (HUST), Wuhan, China. He works at School of Energy and Power Engineering and Wuhan National Laboratory for Optoelectronics in HUST. He received his Ph.D in 2002 from Tsinghua University, China. From 2002 to 2005, he worked in Samsung Advanced Institute of Technology (SAIT) in Korea as a senior engineer and obtained SAIT Best Researcher Award in 2003. In September 2005, he was back to China and became an associate professor. In November 2007, he became a full professor after exceptional promotion. His main research interests are LED and electronics packaging, especially in thermal packaging. He has published more than 60 technical papers, applied and owned 40 patents in USA, Japan, Korea, Europe and China.

Sheng Liu is a Cheung Kong scholar professor of Mechanical Engineering at Huazhong University of Science and Technology and he has a dual appointment at Wuhan National Laboratory for Optoelectronics. He once was a tenured faculty at Wayne State University. He has over 20 years experience in LED/MEMS/IC packaging. He once won prestigious White House/NSF Presidential Faculty Fellow Award in 1995. He obtained his Ph.D from Stanford University in 1992. He is an ASME Fellow. He has filed and owned more than 80 patents in China and USA, and has published more than 300 technical articles, edited more than 9 proceedings in English for ASME and IEEE. 